\title{
Application of trans-sutural distraction osteogenesis based on an optical surgical navigation system to correct midfacial dysplasia
}

ZhenMin Zhao ( $\sim$ zhaozhenmin0098@vip.sina.com )

Peking University Third Hospital

\section{YuJie Chen}

Peking University Third Hospital

ShanShan Du

Peking University Third Hospital

ZhiYu Lin

Peking University Third Hospital

PeiYang Zhang

Peking University Third Hospital

XinLing Zhang

Peking University Third Hospital

JunCheng Wang

Beihang University

\section{Research Article}

Keywords: Optical Surgical Navigation System, midfacial hypoplasia, trans-sutural distraction osteogenesis

Posted Date: February 28th, 2022

DOI: https://doi.org/10.21203/rs.3.rs-1324858/v1

License: (c) (i) This work is licensed under a Creative Commons Attribution 4.0 International License. Read Full License 


\section{Abstract}

Midfacial hypoplasia is a common maxillofacial deformity in patients with cleft lip and palate, which requires surgical treatment. However, achieving noninvasiveness in trans-sutural distraction osteogenesis (TSDO) for midfacial hypoplasia has been difficult. This study aimed to introduce the application of an optical surgical navigation system (OSNS) developed by the authors in TSDO. Six consecutive patients with midfacial hypoplasia (five boys and one girl, with an average age of 10 years; five patients with postoperative cleft lip and palate, and one patient without combined cleft lip and palate) who required TSDO were included in the study. Preoperatively, head computed tomography was performed, and the data were imported into the computer's Mimics software (version 20.0) to design a three-dimensional simulation of the surgical approach. TSDO was performed with the use of the OSNS, and the accuracy and results of the procedure were initially evaluated by comparing the preoperative and postoperative periods. The surgical procedure was successful, with a postoperative follow-up of 4-5 months. All patients demonstrated good treatment results, without complications. Therefore, the OSNS can safely and effectively guide the TSDO technique, essentially correcting midfacial dysplasia, achieving noninvasiveness, improving surgical precision, reducing bleeding, and obtaining better clinical results.

\section{Introduction}

Midfacial hypoplasia is a common maxillofacial deformity in clinical practice, which is mostly observed in patients with cleft lip and palate. In patients with cleft lip and palate, maxillary development is restricted due to surgical or non-surgical infections, scars, and internal underdevelopment, whereas in patients without cleft lip and palate, it is mostly caused by genetic and chromosomal problems, such as the Crouzon syndrome and Apert syndrome ${ }^{1}$. The restricted midfacial development can affect a patient's appearance, pronunciation, and breathing, which could influence the patient's self-esteem ${ }^{2}$. Moreover, it could cause great distress to the patient and family members and is not conducive to a patient's growth and development. Therefore, surgical treatment is performed in patients with midfacial hypoplasia. This has always been the direction of research on maxillofacial surgery ${ }^{3}$. As early as the 1990 s, traditional orthognathic surgical techniques or osteotomy techniques were used, which often required a combination of Lefort osteotomy. This type of osteotomy requires exposing the maxilla and breaking the bilateral maxilla to achieve the effect of advancement, which is highly traumatic and risky ${ }^{4}$. In 1973 , Snyder et al. ${ }^{5}$ applied distraction osteogenesis to the craniofacial skeletal system for the first time. They discovered that the mandible of a dog could be lengthened by distraction and also observed the effect of distraction on craniofacial morphology with more satisfactory results. In 1997, Figueroa et al. ${ }^{6}$ reviewed previous studies and designed an externally adjustable rigid retraction device based on the original device, through which it could be used to treat patients with midfacial hypoplasia in clinical practice with satisfactory results; however, the patients also required the use of combined osteotomy techniques. In 2005, Chunming Liu et al. $7 ; 8$ introduced, for the first time, a minimally invasive, non-osteotomy trans-sutural distraction osteogenesis (TSDO), a traction device that can effectively correct midfacial dysplasia while avoiding the sequelae associated with osteotomy. Subsequently, Zhao Zhenmin et al. ${ }^{9-11}$ have promoted 
this TSDO technique in the clinical practice for over ten years, and achieved satisfactory and long-term follow-up results. TSDO is a safe and reliable technique for midfacial hypoplasia correction ${ }^{12}$. In 2020 , Jiang et al. ${ }^{13}$ conducted a meta-analysis of previous studies and reported that TSDO had a significantly lower recurrence rate than traditional osteotomy distraction. The same results were obtained by Ahn et al. 14 in 2021. TSDO is the preferred treatment for patients with corrected midfacial dysplasia. However, achieving complete noninvasiveness with TSDO has been difficult, and patients often require a small intraoral and nasal basal opening to define the surgical path through a minimally invasive opening. Moreover, revealing the root associated with the tooth within the maxilla has been challenging, even when the maxilla is exposed, which has been difficult to overcome with TSDO.

With the rapid development of information technology, many operations can be made intelligent with the help of computer-aided surgery (CAS) ${ }^{15} ;{ }^{16}$. Here, TSDO was combined with the optical surgical navigation system (OSNS) for the first time to display the surgical situation dynamically in real time, guide intraoperative operations, and predict postoperative results. This greatly increases the accuracy and reduces the trauma associated with surgery ${ }^{17}$. TSDO in combination with CAS can reduce bleeding and collateral damage, offering precise medicine and maximum patient protection.

\section{Results}

Altogether, six patients were included in this study: five boys and one girl, with a mean age of 10 years. Five patients had midfacial dysplasia combined with cleft lip and palate postoperatively, and one patient had no combined cleft lip and palate. The surgical procedure was smooth, the median intraoperative bleeding was $10 \mathrm{ml}$, and the postoperative follow-up was 4-5 m. All patients demonstrated good therapeutic effects without any complications and were satisfied with the results of the postoperative traction. After traction, using the data in the sagittal position, the maxillary deformity was corrected, and the correction of the maxillary deformity was also found in the profile.

\section{Discussion}

The treatment of midfacial hypoplasia is of great significance in adolescents, with the goal of correcting midfacial deformities, improving appearance, articulation and breathing disorders, and regaining selfconfidence ${ }^{2}$. Traditional orthognathic surgery, osteotomy, and traction surgery often require the use of Lefort osteotomy techniques, which are traumatic and highly risky ${ }^{4}$. Hence, they are gradually discontinued in clinical practice for correcting facial hypoplasia in adolescents. Meanwhile, TSDO is a minimally invasive, non-osteotomy procedure that has become the procedure of choice for children with midfacial hypoplasia ${ }^{18}$; ${ }^{19}$; however, a minimally invasive incision is often required to expose the maxilla and define the surgical path to allow observation of the situation related to the roots and nerves in the maxilla, which may damage the roots and nerves in complicated cases. 
Artificial intelligence can be used to assist surgery in other parts of the body to achieve better results ${ }^{20-}$ 22. For example, traditional craniofacial intelligent navigation often requires a positioning device in the head, which can interfere with the placement of the head frame in TSDO and readjustment of the site after intraoperative head position changes. However, this device cannot be sufficiently adjusted at any time, thereby greatly limiting its use in TSDO.

Currently the OSNS is a surgically assisted navigation device that has gained widespread interest owing to its enhanced ability to visualize surgery-related dynamics in real time ${ }^{23}$. In this study, we present the application of an OSNS developed by the authors in TSDO. It has the following advantages: first, it is based on an optical navigation system, which was successfully applied for the first time in TSDO; second, by perfecting the modeling in advance and producing visualized images, it can guide the surgical approach dynamically in real time, which is more minimally invasive and achieves precise medical treatment; third, by combining the use of preoperative data, it allows observation of the situation related to the roots in the maxilla in real time, which minimizes the damage to the roots and teeth; fourth, the OSNS, which no longer requires a frontal fixed signal receiver, can greatly increase the scope of plastic surgery through the positioning kit out of the braces, and can control plastic surgery more precisely; fifth, the 3D Mimics 20.0 software can be used to determine the clinical effect before and after traction, and precisely define the traction site to provide more precise guidance for subsequent research $23 ; 24$.

In this group of six patients, TSDO was performed with the assistance of optical surgery. The operation was successfully performed in all the patients, avoiding incisions through the mucosa and the nasal base of the oral cavity, realizing true "minimally invasiveness," reducing bleeding, and maximizing the protection of the patient's roots and nerves, especially in difficult patients. Preliminary clinical applications have shown that TSDO combined with an OSNS can precisely correct midfacial hypoplasia.

Regarding application, according to different individuals, the design can be tailored to choose the most suitable surgical access. For the surgeon, this combination breaks the dilemma that traditional TSDO is unable to observe the surgical approach dynamically in real time without incision; for the patient, the surgical design is more reasonable, more effective in avoiding other structures in the maxilla, and precise placement of the traction hook can achieve the best traction effect, allowing the patient to obtain the best surgical results with minimal trauma. Therefore, combination with the OSNS accelerates the promotion and application of TSDO-related surgery and further shortens the learning curve ${ }^{25} ;{ }^{26}$, which is another milestone in the field of treatment related to midfacial hypoplasia correction.

The application of an OSNS in TSDO can provide surgeons with better control over surgery, which can effectively improve the efficiency and safety of surgery and reduce patient trauma. However, this study had some limitations. First, surgery time in the early stage of learning combined with artificial intelligence may be slightly longer, although the surgery time can be shortened after improving surgery skills, which is also in line with the traditional learning curve. Second, the sample size was small because the combination of the OSNS and TSDO is the first of its kind. Nevertheless, we intend to expand the sample 
size and combine it with prospective longitudinal clinical studies in the future to obtain more effective results and explore the advantages of TSDO combined with the OSNS.

In conclusion, the OSNS can effectively guide the application of TSDO in correcting midfacial dysplasia. The procedure is non-invasive, improves surgical precision, reduces bleeding, and obtains better clinical results.

\section{Methods}

\section{General information}

Six patients with midfacial hypoplasia who were admitted to the Department of Plastic Surgery, Peking University Third Hospital from July 2021 to August 2021 were enrolled in this study. General indicators, such as patient age, sex, operation method, and bleeding volume, were collected. The patient and demographic characteristics of the patients are summarized in Table 1. All medical protocols in this study adhered to the Declaration of Helsinki. This study was approved by the Institutional Review Board of Peking University Third Hospital (approval number: M2021645). All methods were performed in accordance with the relevant guidelines and regulations of our hospital. Informed consent to release information and images from online open-access publications was obtained from all participants. The following workflow was applied by surgeons and mechanical engineers, as shown in Figure 1.

\section{Procedure}

\section{Step 1: pre-operative preparation}

Preoperatively, a dual-source computed tomography (CT) scanner (SOMATOM Definition Flash 128 Rao, Siemens, Germany) was used to perform a spiral scan of the patient's whole skull with a layer thickness of $0.5 \mathrm{~mm}$, and the CT data were imported into the Mimics 20.0 software in DICOM format data format for craniomaxillofacial 3D reconstruction. Model 3D printing was performed using a Stratasys, Objet 500 Connex 3 3D printer, and the pre-designed surgical design, which is the traction hook placement perforation points, was reflected on the printed model.

\section{Step 2: components and working principle}

The OSNS was based on the following components: binocular navigators, monitors, marker positioning devices, braces, bridging sleeves, and real-time probe devices. The principle is to use intraoperative realtime tracking and positioning technology to calculate the position of the surgical operation target point in relation to the surgical instruments or probes in real time, render them in real time, and then display the model and surgical instruments or probes on the monitor simultaneously to provide accurate surgical 
guidance to the surgeon. The system hardware includes several parts, such as a binocular lens, computer host, and monitor used to identify and position the marker. The alignment simulation was performed on the 3D printed model through an OSNS to test the alignment accuracy. The cranial model is recognized by the binocular lens through the marker on the brace struts and on the surgical instruments, and the image is transmitted to the computer software in real time, which facilitates the simultaneous observation of the operation through the monitor. The alignment accuracy error was $<2 \mathrm{~mm}$ by more than five simulations preoperatively (Fig. 2).

\section{Step 3: OSNS for intraoperative guidance}

Figure 3 illustrates the real-time process of guiding the surgery, dynamically based on the OSNS.

During the operation, this navigation system was used to locate the maxilla at the outer edge of the bony pyriform foramen on both sides, and the surgical access was clearly defined at a distance of $6 \mathrm{~mm}$ above the root of the cuspid and approximately $1 \mathrm{~cm}$ from the lateral edge of the pyriform foramen. With the assistance of the OSNS, the surgeon could observe the real-time location of the probe through the display screen without cutting the upper lip mucosa, separating the tissues to expose the maxilla, and then mechanically punching a hole and placing the retraction hook after the clear location. This greatly reduces soft tissue damage, while protecting the roots of the teeth. For the placement of the cephalic frame, it can be positioned by precise measurement preoperatively, and the position of the fixed screw punching can be reflected in the scalp projection point in real time through the OSNS intraoperatively to avoid visual errors.

\section{Step 4: evaluating the safety and effectiveness of the OSNS}

Postoperatively, a cranial X-ray was performed to clarify the position of the traction hook and calculate the bleeding volume. After traction, the head frame was removed. Cranial CT was reviewed to evaluate the traction effect. The preoperative 3D reconstructed image was used as the base coordinate system, and the 3D cranial model was reconstructed after treatment (after removal of the traction hook), fitted to the base coordinate system to measure the changes in the morphological position of the patient's maxilla before and after treatment and to verify the relationship between the preoperative desired traction hook placement points and the actual postoperative position points to verify the accuracy of the optical navigation system. The coordinates of the bilateral traction hook placement points were measured in 3D space before and after treatment in two patients, as shown in Figure 4, and the data obtained are summarized in Table 2.

\section{Brief steps of the operation}


The patient was in supine, and braces were fitted. After administration of anesthesia, the nasal and oral cavities were routinely disinfected and rinsed with a diluted iodophor. The preoperatively designed surgical access was initially defined using the OSNS for positioning. Along the mark toward the pearshaped foramen in the nasal aperture, mechanical perforation was performed using a K-wire to avoid the root of the tooth. After the successful perforation, retraction hooks were placed through the nostrils. The contralateral side was treated similarly. Using the OSNS to locate the precise site of cephalic brace placement on both sides of the temporal top, an incision of approximately 4-5 $\mathrm{mm}$ in length was made along the direction of the superficial temporal artery terminal alignment using a Melan marker, the scalp was incised. Vascular forceps were used to bluntly separate the subcutaneous to the periosteum, and the cephalic brace was fixed using titanium nails, while the vertical and horizontal rods of the traction brace were installed.

\section{Declarations}

\section{Acknowledgment}

We would like to thank Editage (www.editage.cn) for English language editing.

\section{Author contributions}

Concept and Design: YuJie Chen, ShanShan Du, JunCheng Wang, ZhenMin Zhao

Collection and assembly of data: YuJie Chen, ShanShan Du, ZhiYu Lin, PeiYang Zhang, XinLing Zhang

Data analysis and interpretation: YuJie Chen, ShanShan Du,

Manuscript writing: All authors

Final approval of manuscript: All authors

\section{Data availability statement}

The datasets developed and analyzed during this study are available from the corresponding author on reasonable request. All datasets generated for this study are included in the article, Patient names are inconvenient to provide because of the need to protect clinical patient privacy.

\section{Competing interests statement}

The authors declare no competing interests.

\section{References}


1. Gao,F. Clinical and Experimental Studies on the Trans-sutural Distraction Osteogenesis., Vol. Doctor: Peking Union Medical College Hospital, P. 72, (2012).

2. Rachmiel, A., Aizenbud, D. \& Peled, M. Long-Term Results in Maxillary Deficiency Using Intraoral Devices. Int J Oral Maxillofac Surg. 34, 473-479 (2005).

3. Figueroa, A. A., Polley, J. W., Friede, H. \& Ko, E. W. Long-Term Skeletal Stability After Maxillary Advancement with Distraction Osteogenesis Using a Rigid External Distraction Device in Cleft Maxillary Deformities. Plast. Reconstr. Surg. 114, 1382-1392, 1393-1394 (2004).

4. Molina, F., Ortiz, M. F., de la Paz, A. M. \& Barrera, J. Maxillary Distraction: Aesthetic and Functional Benefits in Cleft Lip-Palate and Prognathic Patients During Mixed Dentition. Plast. Reconstr. Surg. 101, 951-963 (1998).

5. Snyder, C. C., Levine, G. A., Swanson, H. M. \& Browne, E. J. Mandibular Lengthening by Gradual Distraction. Preliminary Report. Plast. Reconstr. Surg. 51, 506-508 (1973).

6. Polley, J. W. \& Figueroa, A. A. Management of Severe Maxillary Deficiency in Childhood and Adolescence through Distraction Osteogenesis with an External, Adjustable, Rigid Distraction Device. J. Craniofac. Surg. 8, 181-185, 186 (1997).

7. Min, Hou.Chun-ming \Liu.Li-min, Liang et al. Sutural Distraction Osteogenesis for Correction of Midface Hypoplasia in Patients with Cleft Lip and Palate. Journal of Oral and Maxillofacial Surgery 15, 267-270 (2005).

8. Chun-ming, Liu.Xu-ming, Huang.Min, Hou, et al. Trans-sutural distraction osteogenesis for early correction of midfacial hypoplasia in children: a primary clinical report. Chinese Journal of Plastic Surgeru. 21, 90-93 (2005).

9. Tong, H. et al. Transsutural Distraction Osteogenesis Applied to Maxillary Complex with New Internalized Distraction Device: Analysis of the Feasibility and Long-Term Osteogenesis Outcome. J. Craniofac. Surg. 26, 402-407 (2015).

10. Tong, H. et al. Trans-Sutural Distraction Osteogenesis for Midfacial Hypoplasia in Growing Patients with Cleft Lip and Palate: Clinical Outcomes and Analysis of Skeletal Changes. Plast. Reconstr. Surg. 136, 144-155 (2015).

11. Tong, H. et al. Three-Dimensional Quantitative Evaluation of Midfacial Skeletal Changes After TransSutural Distraction Osteogenesis for Midfacial Hypoplasia in Growing Patients with Cleft Lip and Palate. J Craniomaxillofac Surg. 43, 1749-1757 (2015).

12. Tong, H.Trans-sutural Distraction Osteogenesis for Midfacial Hypoplasia in Growing Patients with Cleft Lip and Palate: Study on the Mechanism and Long-Term Skeletal Stability., Vol.Doctor: Peking Union Medical College Hospital, 2016.

13. Jiang, L. et al. Relapse Rate After Surgical Treatment of Maxillary Hypoplasia in Non-Growing Cleft Patients: A Systematic Review and Meta-Analysis. Int J Oral Maxillofac Surg. 49, 421-431 (2020).

14. Ahn, H. W., Kim, S. J. \& Baek, S. H. Miniplate-Anchored Maxillary Protraction in Adolescent Patients with Cleft Lip and Palate: A Literature Review of Study Design, Type and Protocol, and Treatment Outcomes. Orthod. Craniofac. Res. 24 Suppl 1, 21-30 (2021). 
15. Joskowicz, L. Computer-Aided Surgery Meets Predictive, Preventive, and Personalized Medicine. EPMA J. 8, 1-4 (2017).

16. Edwards, S. P. Computer-Assisted Craniomaxillofacial Surgery. Oral Maxillofac Surg Clin North Am. 22, 117-134 (2010).

17. Ma, C., Chen, G., Zhang, X., Ning, G. \& Liao, H. Moving-Tolerant Augmented Reality Surgical Navigation System Using Autostereoscopic Three-Dimensional Image Overlay. IEEE J Biomed Health Inform. 23, 2483-2493 (2019).

18. Tong, H. et al. Trans-Sutural Distraction Osteogenesis for Midfacial Hypoplasia in Growing Patients with Cleft Lip and Palate: Clinical Outcomes and Analysis of Skeletal Changes. Plast. Reconstr. Surg. 136, 144-155 (2015).

19. Tong, H. et al. Transsutural Distraction Osteogenesis Applied to Maxillary Complex with New Internalized Distraction Device: Analysis of the Feasibility and Long-Term Osteogenesis Outcome. J. Craniofac. Surg. 26, 402-407 (2015).

20. Cho, H. S. et al. Can Augmented Reality be Helpful in Pelvic Bone Cancer Surgery? An in Vitro Study. Clin Orthop Relat Res. 476, 1719-1725 (2018).

21. Guha, D. et al. Augmented Reality in Neurosurgery: A Review of Current Concepts and Emerging Applications. Can. J. Neurol. Sci. 44, 235-245 (2017).

22. Xia, J. J. et al. Accuracy of the Computer-Aided Surgical Simulation (Cass) System in the Treatment of Patients with Complex Craniomaxillofacial Deformity: A Pilot Study. J Oral Maxillofac Surg. 65, 248-254 (2007).

23. Ma, C., Chen, G., Zhang, X., Ning, G. \& Liao, H. Moving-Tolerant Augmented Reality Surgical Navigation System Using Autostereoscopic Three-Dimensional Image Overlay. IEEE J Biomed Health Inform. 23, 2483-2493 (2019).

24. Ewurum, C. H., Guo, Y., Pagnha, S., Feng, Z. \& Luo, X. Surgical Navigation in Orthopedics: Workflow and System Review. Adv. Exp. Med. Biol. 1093, 47-63 (2018).

25. Kluger, M. D., Vigano, L., Barroso, R. \& Cherqui, D. The Learning Curve in Laparoscopic Major Liver Resection. J Hepatobiliary Pancreat Sci. 20, 131-136 (2013).

26. Pernar, L. et al. An Appraisal of the Learning Curve in Robotic General Surgery. Surg. Endosc. 31, 4583-4596 (2017).

\section{Tables}

Table.1

Characteristics of the 6 patients included in the study 


\begin{tabular}{|c|c|c|c|c|c|c|c|c|}
\hline Patient & $\begin{array}{l}\text { Age } \\
\text { (years } \\
\text { old) }\end{array}$ & Sex & Disease & $\begin{array}{l}\text { Cleft lip } \\
\text { and } \\
\text { palate }\end{array}$ & Complication & $\begin{array}{l}\text { Time } \\
\text { (hours) }\end{array}$ & $\begin{array}{l}\text { Bleeding } \\
(\mathrm{ml})\end{array}$ & $\begin{array}{l}\text { Follow- } \\
\text { up period } \\
\text { 『months } \rrbracket\end{array}$ \\
\hline 1 & 7 & $M$ & $\begin{array}{l}\text { maxillary } \\
\text { retrusion }\end{array}$ & $Y$ & $\mathrm{~N}$ & 5.5 & 10 & 5 \\
\hline 2 & 10 & $\mathrm{M}$ & $\begin{array}{l}\text { maxillary } \\
\text { retrusion }\end{array}$ & Y & $\mathrm{N}$ & 7 & 5 & 5 \\
\hline 3 & 9 & $M$ & $\begin{array}{l}\text { maxillary } \\
\text { retrusion }\end{array}$ & $Y$ & $\mathrm{~N}$ & 7 & 5 & 4 \\
\hline 4 & 12 & $\mathrm{M}$ & $\begin{array}{l}\text { maxillary } \\
\text { retrusion }\end{array}$ & Y & $\mathrm{N}$ & 6.5 & 10 & 4 \\
\hline 5 & 9 & $M$ & $\begin{array}{l}\text { maxillary } \\
\text { retrusion }\end{array}$ & Y & $\mathrm{N}$ & 6 & 10 & 5 \\
\hline 6 & 13 & $\mathrm{~F}$ & $\begin{array}{l}\text { maxillary } \\
\text { retrusion }\end{array}$ & $\mathrm{N}$ & $\mathrm{N}$ & 4 & 5 & 4 \\
\hline
\end{tabular}

Table.2

Three-dimensional spatial position and SNA and SNB angles of the preoperative and postoperative traction hook placement points. 


\begin{tabular}{|c|c|c|c|c|c|c|c|c|c|}
\hline \multirow[b]{2}{*}{ Patient } & \multirow[b]{2}{*}{ Operation } & \multicolumn{2}{|c|}{ X (Transverse) } & \multicolumn{2}{|c|}{ Y (sagittal) } & \multicolumn{2}{|c|}{ Z (coronal) } & \multirow[t]{2}{*}{ SNA } & \multirow[t]{2}{*}{ SNE } \\
\hline & & A (left) & B (right) & $A$ (left) & B (right) & $A(l e f t)$ & B (right) & & \\
\hline \multirow[t]{2}{*}{1} & pre- & 67.5 & 64.5 & 132.7 & 102.7 & 189.4 & 196,2 & $69^{\circ}$ & $71^{\circ}$ \\
\hline & post- & 66.5 & 63.5 & 133.8 & 102.2 & 191.8 & 199.1 & $76^{\circ}$ & $71^{\circ}$ \\
\hline \multirow[t]{2}{*}{2} & pre- & 180.5 & 184 & 145.1 & 98.7 & 286.7 & 191.9 & $86^{\circ}$ & $80^{\circ}$ \\
\hline & post- & 179.5 & 183.5 & 145.6 & 98.3 & 193.8 & 197.1 & $97^{\circ}$ & $82^{\circ}$ \\
\hline \multirow[t]{2}{*}{3} & pre- & 95.5 & 93.5 & 124.1 & 83.6 & 161.8 & 164.3 & $87^{\circ}$ & $77^{\circ}$ \\
\hline & post- & 95 & 93 & 125 & 84 & 167.7 & 169 & $95^{\circ}$ & $78^{\circ}$ \\
\hline \multirow[t]{2}{*}{4} & pre- & 83 & 81 & 137 & 95 & 174 & 178 & $85^{\circ}$ & $86^{\circ}$ \\
\hline & post- & 83 & 80 & 138 & 95.3 & 178.2 & 183 & $93^{\circ}$ & $86^{\circ}$ \\
\hline \multirow[t]{2}{*}{5} & pre- & 98 & 95 & 145.1 & 108.7 & 158.7 & 159.9 & $83^{\circ}$ & $85^{\circ}$ \\
\hline & post- & 98 & 94 & 144.8 & 107.7 & 164.6 & 164.2 & $92^{\circ}$ & $86^{\circ}$ \\
\hline \multirow[t]{2}{*}{6} & pre- & 84 & 84.5 & 158.4 & 122 & 192.6 & 189.1 & $84^{\circ}$ & $83^{\circ}$ \\
\hline & post- & 83.5 & 84 & 158.6 & 122.3 & 197.8 & 194.1 & $90^{\circ}$ & $84^{\circ}$ \\
\hline $\begin{array}{l}\text { It can b } \\
\text { bilatera } \\
\text { the corc } \\
\text { SNA an } \\
\text { maxilla }\end{array}$ & $\begin{array}{l}\text { seen that th } \\
\text { raction poir } \\
\text { al axis, indi } \\
\text { SNB angle } \\
\text { nd no signif }\end{array}$ & $\begin{array}{l}\text { asurem } \\
\text { ant char }\end{array}$ & $\begin{array}{l}\text { xillary t } \\
\text { sverse a } \\
\text { ficant al } \\
\text { ts, it can } \\
\text { in the }\end{array}$ & $\begin{array}{l}\text { so be se } \\
\text { idible, w }\end{array}$ & $\begin{array}{l}\text { y, there is } \\
\text { tal axis, } \\
\text { ee maxill } \\
\text { that ther } \\
\text { significa }\end{array}$ & $\begin{array}{l}\text { ttle char } \\
t \text { there is } \\
\text { By preop } \\
\text { s a sign } \\
\text { improv }\end{array}$ & $\begin{array}{l}\text { in the po } \\
\text { significan } \\
\text { ative and } \\
\text { ant anteri } \\
\text { ent in the }\end{array}$ & $\begin{array}{l}\text { tion o } \\
\text { differ } \\
\text { stop } \\
\text { ity of } \\
\text { nderb }\end{array}$ & $\begin{array}{l}\text { he } \\
\text { ce to } \\
\text { tive } \\
\text { e }\end{array}$ \\
\hline
\end{tabular}

Figures 


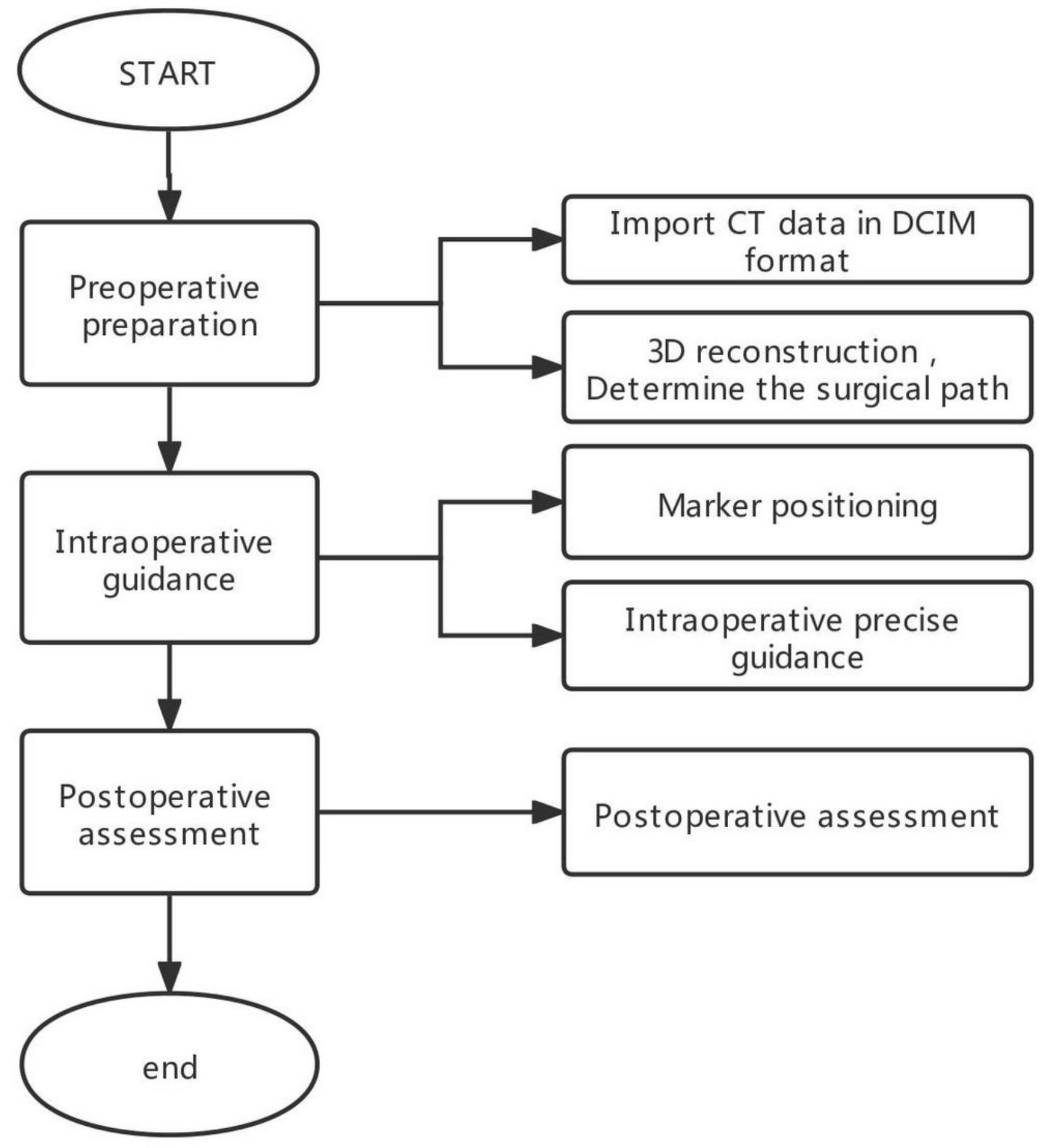

Figure 1

The workfow of the whole process. 

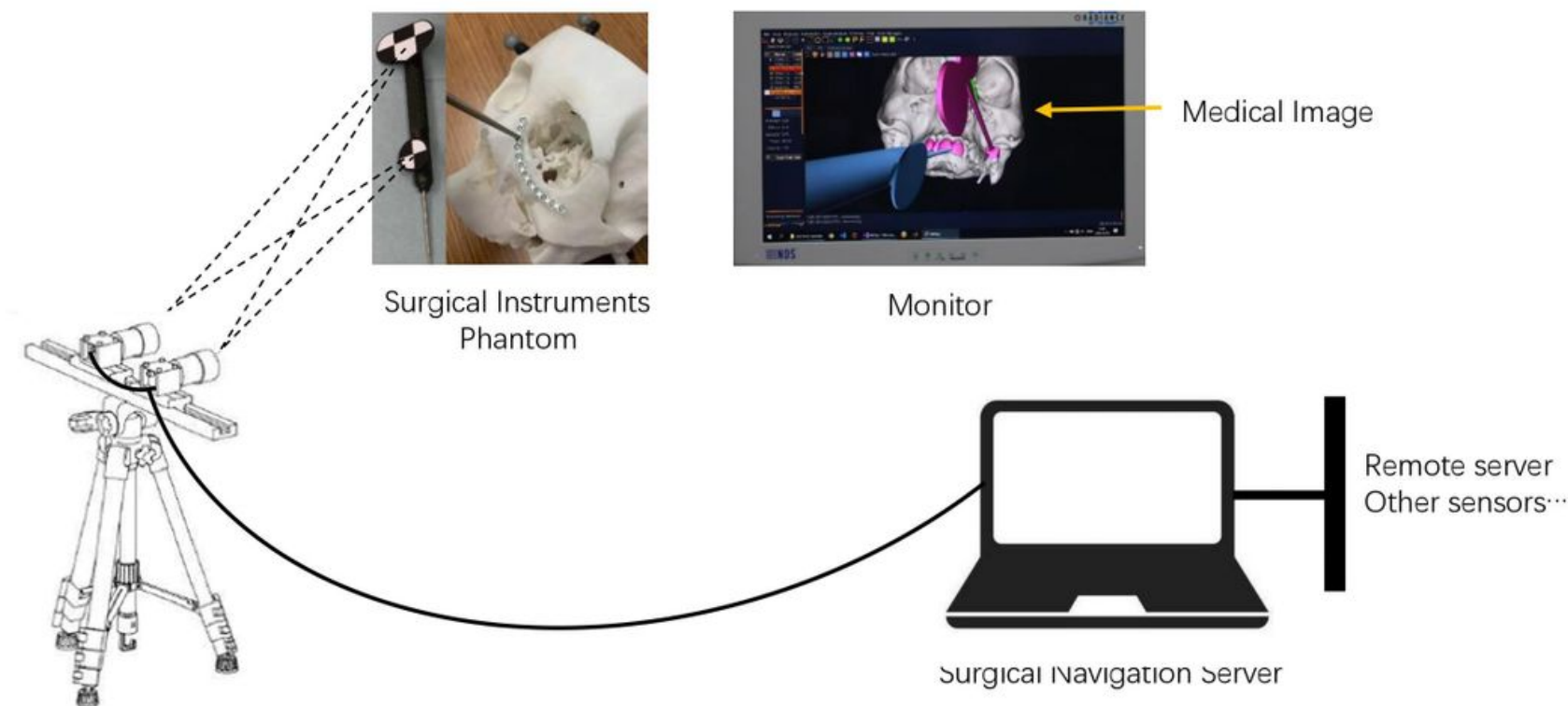

Location tracking device

\section{Figure 2}

Components and working principle of optical navigation system.

Identification of the probe and the Marker on the braces by the binocular lens for positioning on the model, while the probe and the reconstructed model of the skull are presented in real time on the monitor. 

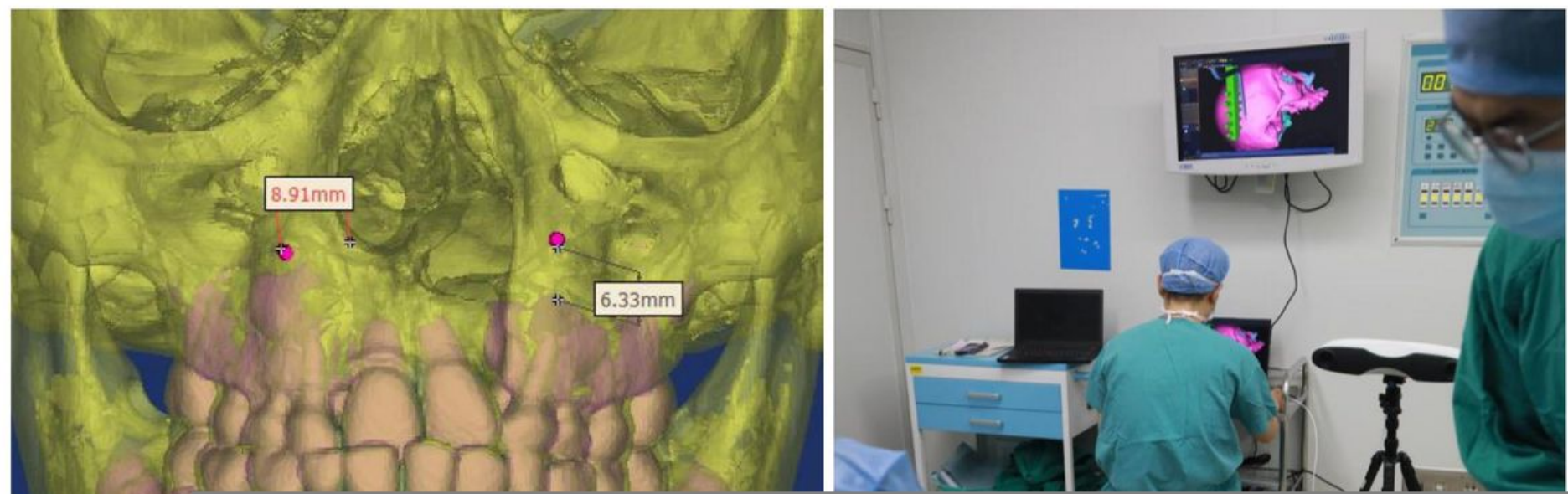

\section{Figure 3}

Illustrates the real-time process of guiding the surgery, dynamically based on this navigation system.

a, Preoperative design of traction hook placement points; $b$, the position of the fixed screw punching can be reflected in the scalp projection point in real time through the OSNS intraoperatively; c,d, With the assistance of the navigation system, the surgeon could observe the real-time location of the probe through the display screen without cutting the upper lip mucosa, separating the tissues to expose the maxilla. 

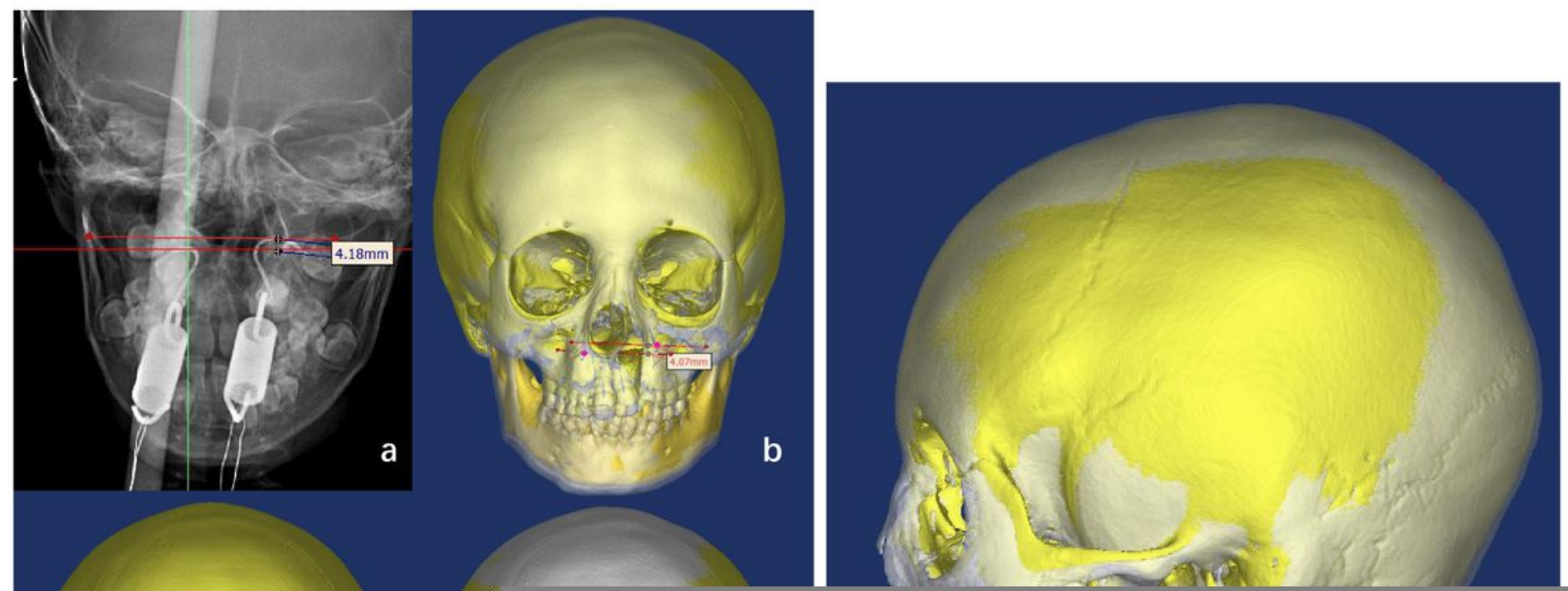

\section{Figure 4}

Evaluating the safety and effectiveness of the OSNS.

a, A cranial X-ray was performed to clarify the position of the traction hook;

$b, c, d$, The preoperative 3D reconstructed image was used as the base coordinate system, and the 3D cranial model was reconstructed after treatment (after removal of the traction hook), fitted to the base coordinate system to measure the changes in the morphological position of the patient's maxilla;

e, The morphological position of the patient's maxilla before and after treatment. 